\title{
Evaluation of the optical switching characteristics of erbium-doped fibres for the development of a fibre Bragg grating sensor interrogator
}

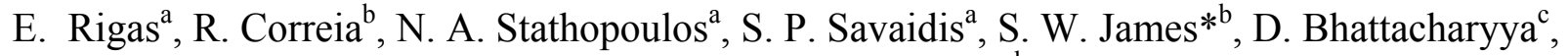 \\ P. B. Kirby ${ }^{c}$ and R. P. Tatam ${ }^{b}$ \\ ${ }^{a}$ Department of Electronic Engineering, Technological Education Institute (TEI) of Piraeus, Greece. \\ ${ }^{\mathrm{b}}$ Engineering Photonics, and ${ }^{\mathrm{c} D e p a r t m e n t}$ of Manufacturing \& Materials, Cranfield University, UK.
}

\begin{abstract}
A polling topology that employs optical switching based on the properties of erbium-doped fibres (EDFs) is used to interrogate an array of FBGs. The properties of the EDF are investigated in its pumped and un-pumped states and the EDFs' switching properties are evaluated by comparing them with a high performance electronically controlled MEM optical switch. Potential advantages of the proposed technique are discussed.
\end{abstract}

Keywords: FBG, optical switching, sensor interrogation, EDF, erbium-doped fibres

\section{INTODUCTION}

Optical fibre Bragg grating (FBG) stress and strain sensors and their corresponding interrogation systems have attracted great interest, mainly due to the ability multiplex an array of sensors within a single length of optical fibre ${ }^{1}$. Wavelength division multiplexing (WDM) is perhaps the most widely employed FBG interrogation technique, requiring the use of often expensive wavelength tuneable components, such as tuneable filters ${ }^{2,3}$ or tuneable lasers ${ }^{4}$ to scan through the optical spectrum, and requiring that the FBG sensors are fabricated with discrete reflection wavelengths. The use of arrays of nominally identical FBGs in spatial- or time-division multiplexed topologies is attractive as it could reduce the cost of the sensor array ${ }^{5,6,7}$ but this can impose more complexity on the rest of the system. Spatially multiplexed topologies require a filter and detector dedicated to each sensor, and the sensor network topology is often lossy, placing demands on the power budget, while time-division-multiplexed approaches require short pulsed sources and fast electronics, and limit the minimum spatial separation of the FBGs in the array. Therefore, there is a motivation for the development of new interrogators, with ease of manufacture at a low cost, by avoiding wavelength division multiplexing and using identical FBG sensors.

Herein, we adopt the topology proposed in [8] which exploits the switching properties of an optically-pumped Erbiumdoped fibre (EDF) to interrogate an FBG sensor array, and we evaluate its experimental implementation. The switching states of an EDF correspond to the un-pumped state, where the EDF behaves as a saturable absorber, and the pumped state, where the EDF behaves as an optical all-pass device. In particular, we propose the use of an FBG interrogator system capable of reading multiple identical FBGs, with multiplexing achieved by using a polling topology which employs optical switching, based on the properties of EDFs. The applicability of this method in a multisensory FBG interrogation is evaluated experimentally and the EDF switch is compared with an electronically controlled optical MEM switch.

\section{EXPERIMENTS \& RESULTS}

The fibre used was Liekki Er110-4/125 (nLigtht Corp), with a nominal attenuation of $110 \mathrm{~dB} \cdot \mathrm{m}^{-1}$. The spectra of the fibre in its pumped state and when pumped at $980 \mathrm{~nm}$ with a power of $100 \mathrm{~mW}$ are shown in figure 1 . The insert in figure 2 shows the configuration of the sensors, which consisted of a shoft length of erbium doped fibre that was spliced in-series with a length of single mode fibre in which an FBG of reflectivity of approximately $25 \%$ had been fabrciated. For clarity, in order to distinguish between the different sensors, FBGs with slightly different reflectances and wavelengths have been used, as shown in figure 2, but the system would operate with identical FBGS.

*s.w.james@cranfield.ac.uk; phone +44 (0) 1234 754623; cranfield.ac.uk

23rd International Conference on Optical Fibre Sensors, edited by José Miguel López-Higuera,

Julian Jones, Manuel López-Amo, José Luis Santos, Proc. of SPIE Vol. 9157, 91571M

(C) 2014 SPIE · CCC code: $0277-786 X / 14 / \$ 18 \cdot$ doi: 10.1117/12.2059607

Please refer to any applicable publisher terms of use

Proc. of SPIE Vol. 9157 91571M-1 


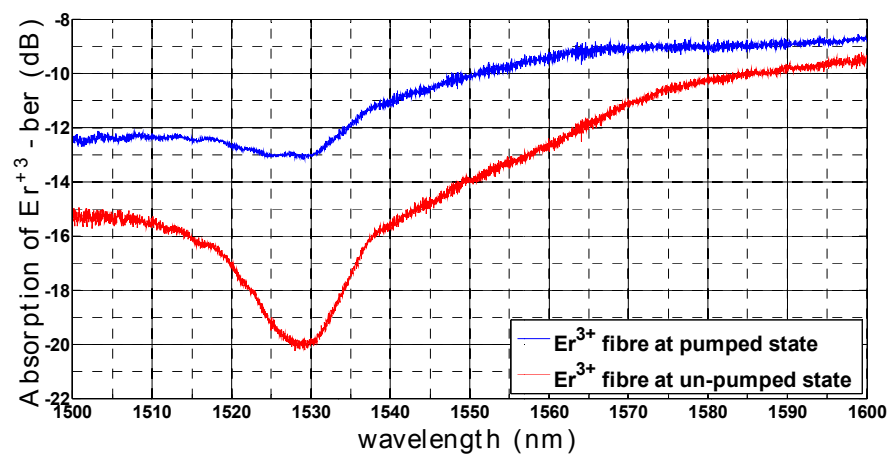

Figure 1. Pumped and un-pumped transmission spectra of the Erbium-doped optical fibre.

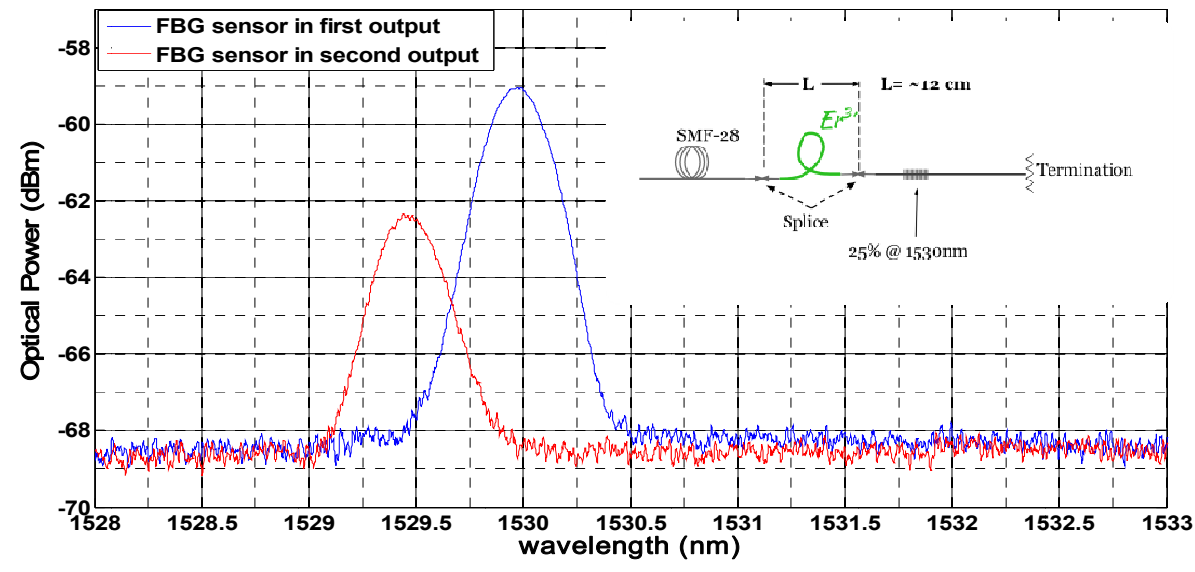

Figure 2. The spectra of the two sensors. The insert shows a schematic of the sensor element configuration.

The first configuration used an all-optical MEM switch to pole the two FBG sensors, with the pump source operating continuously, and is shown in figure 3. This system was used as a reference optical switching topology to aid the evaluation of the EDF optical switching. Figure 4(a) shows the spectrum recorded when the FBG1 was poled, in its relaxed and strained states, while figure 4(b) shows the spectra recorded when the FBG2 was poled. It is evident that the MEM switch provides good isolation between the two branches

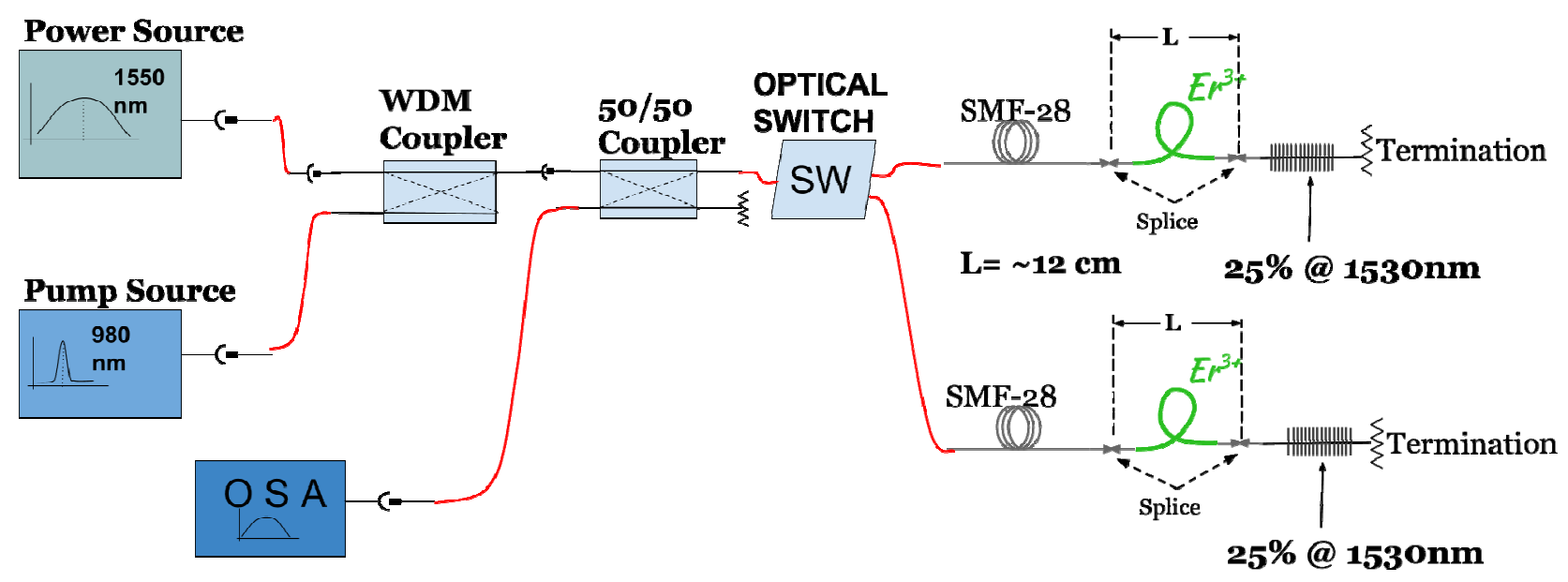

Figure 3. The experimental setup using an MEM switch to pole the FBG sensors. Where OSA is the optical spectrum analyser, 50/50 is a $3 \mathrm{~dB}$ optical coupler, SW is the optical switch and WDM is a wavelength-division multiplexing coupler. 

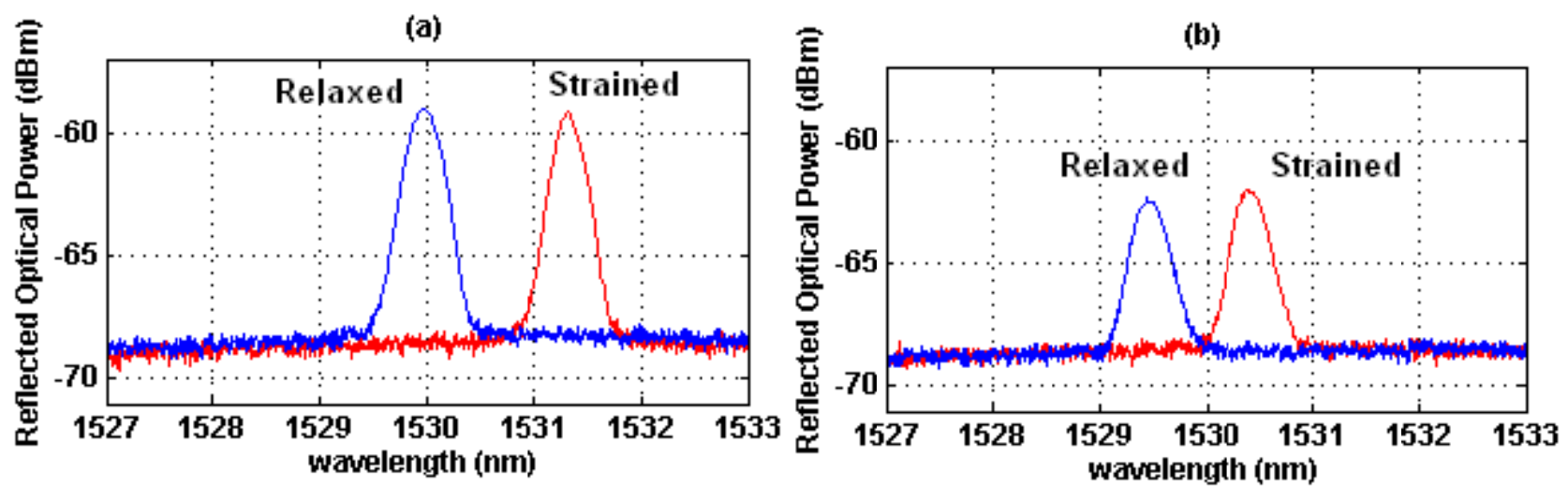

Figure 4. The reflected spectra obtained with the sensors in their relaxed and strained sates when (a) FBG1 and (b) FBG2 were poled using the MEM fibre optic switch

The configuration used for the evaluation of the EDFs' switching properties is shown in figure 5. In this configuration, the pumping sources are switched on and off in order to drive the corresponding EDFs between their pumped and unpumped state. In its un-pumped state, the EDF will absorb the optical reflection from the FBG sensor that is connected in series. As a result, a significant isolation between the two branches is anticipated.

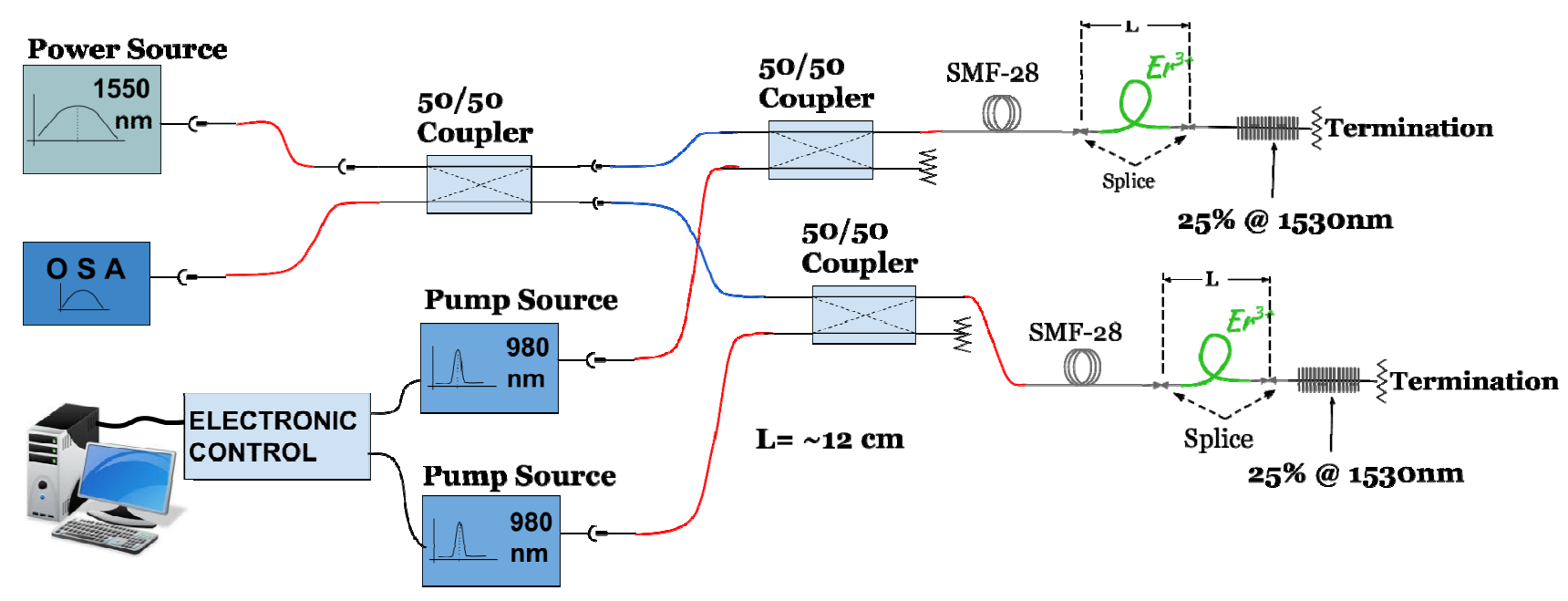

Figure 5. The experimental setup using erbium-doped fibers and switching of the pump sources to pole the FBG sensors. Where OSA is the optical spectrum analyser, $50 / 50$ is a $3 \mathrm{~dB}$ optical coupler.

Figure 6 shows the refection spectra recorded when the test setup of figure 5 was employed Within the limitations of the SNR of the system, it can be seen that the use of EDF optical switching provides the same isolation as that provided by the MEM switch. In figure 4 that the noise floor in the EDF based setup is at $-70 \mathrm{dBm}$ and below, in contrast with the first setup which is about -68 to $-69 \mathrm{dBm}$. 

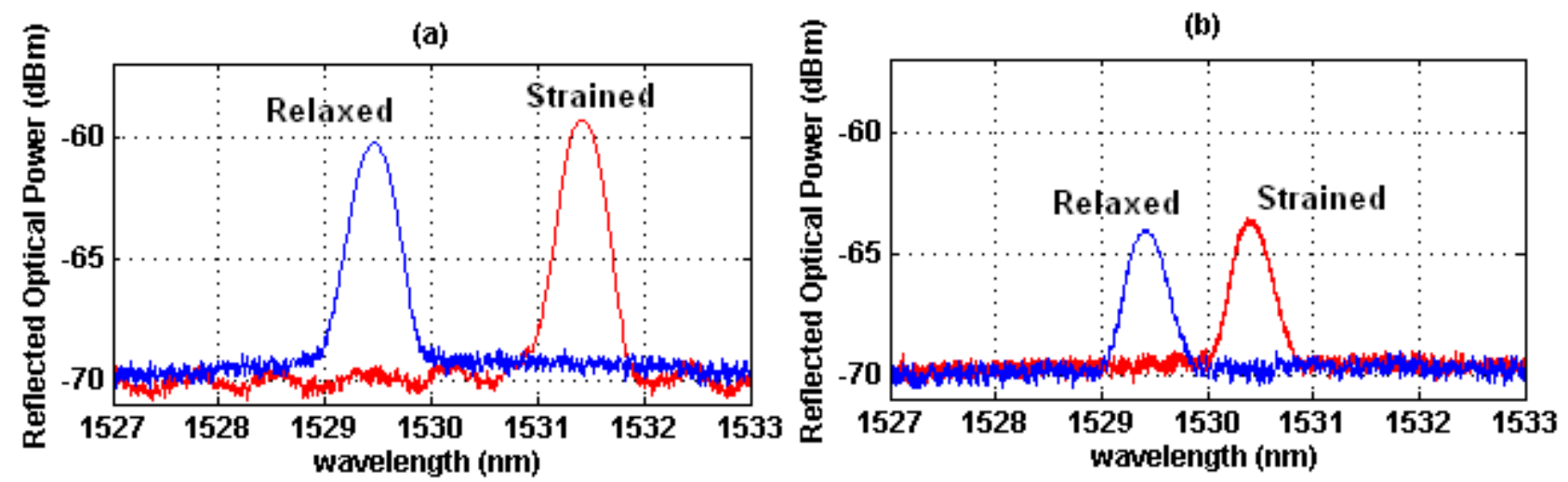

Figure 6. The reflected spectra obtained with the sensors in their relaxed and strained sates when (a) FBG1 and (b) FBG2 were poled by switching pump source power.

\section{CONCLUSIONS}

The use of EDFs as switching components in a multi-sensor interrogation system has been examined experimentally. Commercially available heavily Erbium-doped fibres have been evaluated in their un-pumped state and have been shown to provide attenuation suitable for switch operation. The switching properties of the aforementioned EDFs have been verified experimentally in the pumped and the un-pumped state. As a result, an interrogation scheme utilizing optically switchable FBGs is feasible. A typical single FBG interrogator has been modified in order to expand its operation by multiplexing two FBGs using optically switchable EDFs.

\section{REFERENCES}

[1] B. Lee and J. Yoonchan, Fiber Optic Sensors, 1st ed., F. T. S. Yu and S. Yin, Eds., New York, USA: Marcel Dekker, (2002).

[2] M. D. Todd, G. A. Johnson and B. L. Althouse, "A novel Bragg grating sensor interrogation system utilizing a scanning filter, a Mach-Zehnder interferometer and a 3×3 coupler", Meas.Sci.Technol., 12, 771-777 (2001).

[3] A. D. Kersey, T. A. Berkoff and W. W. Morey, "Multiplexed fiber Bragg grating strain-sensor system with a fiber Fabry--Perot wavelength filter", Opt. Lett., 12, 1370-1372 (1993).

[4] G. Ball, W. Morey and P. Cheo, "Fiber laser source/analyzer for Bragg grating sensor array interrogation", J. Lighwave Technol., 12, 700-703 (1994).

[5] A. Wilson, S. W. James and R. P. Tatam, "Time-division-multiplexed interrogation of fibre Bragg grating sensor using laser diodes", Meas.Sci.Technol., 12, 181-187 (2001).

[6] S. Melle, K. Liu and R. Measures, "A passive wavelength demodulation system for guided-wave Bragg grating sensors", IEEE Photon.Technol.Lett., 4, 516-518 (1992).

[7] C. Caucheteur, K. Chah, F. Lhomme, M. Blondel and P. Megret, "Autocorrelation demodulation technique for fiber Bragg grating sensor", IEEE Photon.Technol.Lett., 16, 2320-2322 (2004).

[8] N.A.Stathopoulos, S. P. Savaidis, H. Simos, M. Rangoussi and P. Kervalishvili, "Simulation and properties of Erbium-Doped Distributed Bragg Reflectors (ED-DBRs) and Fiber Bragg Gratings (ED-FBGs)," Opt.Fib.Technol., 19, 369-377 (2013). 


\section{Evaluation of the optical switching characteristics of erbium-doped fibres for the development of a fibre Bragg grating sensor interrogator}

Rigas, E.

SPIE

Rigas, E. et al. (2014) Evaluation of the optical switching characteristics of erbium-doped fibres for the development of a fibre Bragg grating sensor interrogator, Proceedings of the 23rd international conference on optical fibre sensors (OFS-23), Santander, Spain, 2-5 June 2014, pp91571M-1-4 http://dx.doi.org/10.1117/12.2059607 Downloaded from Cranfield Library Services E-Repository 